\title{
Oceanic Wave Energy Conversion by a Novel Permanent Magnet Linear Generator Capable of Preventing Demagnetization
}

\author{
Omar Farrok, Member, IEEE, Md. Rabiul Islam, Senior Member, IEEE, Md. Rafiqul Islam Sheikh, \\ Member, IEEE, Youguang Guo, Senior Member, IEEE, Jianguo Zhu, Senior Member, IEEE, and Gang \\ Lei, Member, IEEE
}

\begin{abstract}
In the conventional permanent magnet linear generators (PMLGs) used for oceanic wave energy conversion system, demagnetization could cause everlasting degradation in electrical power generation. This paper presents a new design that can be applied to various PMLGs to avoid demagnetization. To check the effectiveness of the proposed technique, a PMLG is considered which allows both the fixed and variable length of air gaps for analysis. The finite element analysis is used by using the software package ANSYS/Ansoft to simulate the testing PMLG for two conditions: with and without using the proposed technique. Different parameters and characteristics of the PMLG under both conditions are presented in detail. Both the simulation and test results show that the proposed design is able to avoid the demagnetization problem successfully.
\end{abstract}

Index Terms-Demagnetization, oceanic wave energy conversion, permanent magnet linear generator, variable length of air gap.

\section{INTRODUCTION}

$\mathrm{E}^{\mathrm{s}}$ LECTRICAL power generation systems from renewable energy resources (RERs) are progressively growing their

Manuscript received November 23, 2017; revised May 12, 2018; accepted July 26, 2018. Date of publication 2018; date of current version , 2018. Paper 2017-EMC-1490.R1, presented at the 2017 IEEE International Conference on Electrical Machines and Systems, Sydney, Australia, August 11-14, and approved for publication in the IEEE TRANSACTIONS ON INDUSTRY APPLICATIONS by the Electric Machines Committee of the IEEE Industry Applications Society.

O. Farrok is with the Department of Electrical and Electronic Engineering, Ahsanullah University of Science \& Technology, Dhaka-1208, Bangladesh, (e-mail: omarruet@gmail.com,omarruet@yahoo.com).

M. R. Islam is with the Faculty of Engineering and Information Sciences, University of Wollongong, New South Wales 2522, Australia (e-mail: rabiulbd@hotmail.com,mrislam@uow.edu.au).

M. R. I. Sheikh is with the Department of Electrical and Electronic Engineering, Rajshahi University of Engineering \& Technology, Rajshahi 6204, Bangladesh (ris_eee@ruet.ac.bd).

Y. G. Guo and G. Lei are with the Faculty of Engineering and Information Technology, University of Technology Sydney, Broadway, New South Wales 2007, Australia (e-mails: youguang.guo-1@uts.edu.au; gang.lei@uts.edu.au).

J. G. Zhu is with the School of Electrical and Information Engineering, University of Sydney, New South Wales 2006, Australia (e-mails: jianguo.zhu@sydney.edu.au).

Color versions of one or more of the figures in this paper are available online at http://ieeexplore.ieee.org.

Digital Object Identifier 10.1109/TIA.2018 coverage in power systems [1]. The most common sources of RERs are the solar and wind energy which are being used either individually [2], [3] or together in hybrid form [4], [5]. Each of this power system may include any type of storage [6], [7] to continue power flow during the absence of one or more of these RERs. The oceanic wave energy (OWE) which is developed rapidly is being considered as a strategic source of renewable energy [8].

The OWE is highly available and predictable compared to other conventional RERs. Solar power is not available at night and the wind flow time is uncertain. Different types of prototype converters of OWE are installed not only in the region of higher altitude such as in European Union but also in some Asian countries such as Japan and India [9]. The linear generators are becoming very popular due to their benefit to connect with direct drive systems for OWE conversion systems. Among different types of OWE converters, various types of permanent magnet linear generators (PMLGs) have been described in [10]-[16]. These conventional PMLGs are designed such that the stator and translator can maintain a constant air gap length. Fig. 1 illustrates a conventional OWE conversion system where a PMLG is connected to a float which is basically a point absorber.

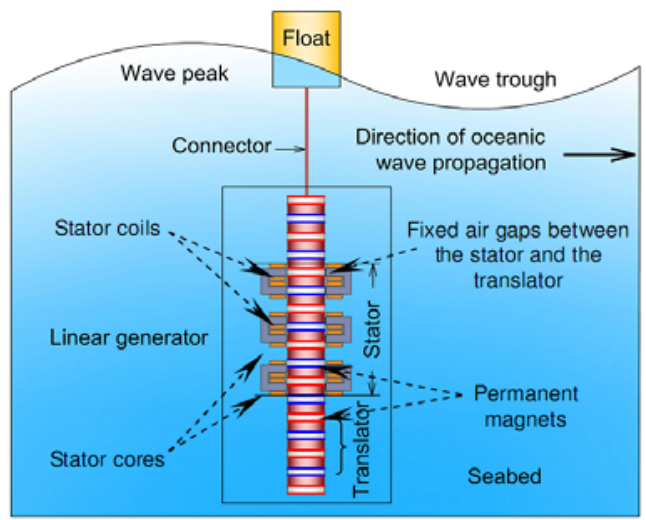

Fig. 1. An existing linear generator with fixed length of air gap.

Various types of PMLGs depending on the physical architecture are being designed and analyzed. Among them, there are two major categories: tubular type [17] and flat type [18]. Generally, the construction of the translator of a PMLG 
is complex because of the coexistence of the permanent magnets and the translator core. As a result, increase in temperature takes place in the translator during its operation which is one of the main reasons of mechanical failure and demagnetization of the permanent magnets [18].

The advantages of linear generators with flat type constructon is pointed out in [19] which is basically a double sided PMLG driven by a point absorber type power take off system. The design of PMLGs is being developed in different ways. A theoretical concept of a new design of the translator of a flat PMLG for OWEC is explained in [20]. Because of variable magnitude and frequency of the oceanic wave the generated voltage and frequency of the PMLG widely changes. For this reason, a controller is necessary to stabilize the output voltage which is generated by the PMLG. A comparison of the generated voltages from a PMLG with and without using a fuzzy logic controller is shown in [21] for the same condition of the oceanic wave.

The nonlinear torque model of the permanent magnet brushless synchronous motor is described in [22] by symbolic regression. The shape optimization is introduced in [23] which is a new concept to be applied on the PMLG for generation of more electricity. It is also shown that less amount of steel core is needed to generate the same amount of electrical power in the same PMLG due to the shape optimization. The finite element based optimization of the three-phase electrical machine is also shown in [24].

Different PMLGs are studied and it is analyzed that demagnetization happens at the time of electrical power generation. The PMLG with variable air gap is introduced in [25] for prevention of demagnetization. The superconductor made of magnesium-diboride is used to make a superconducting linear generator [26] to avoid demagnetization of the flux switching and a Vernier hybrid type PMLG. Two different linear generators using superconductors are presented in [27], [28]. An electromagnetic [29] and a superconducting [30] linear generator for OWE conversion are proposed to prevent demagnetization. The amount of copper loss is high if the linear generator is made by electromagnets. On the other hand, superconducting linear generators are expensive not only for the cost of superconductor itself but also for maintaining cryogenic temperature inside the ocean.

Demagnetization of the permanent magnet is one of the reasons of malfunctioning and performance degrading of the power generation system which are stated in [31], [32]. demagnetization is irreversible according to the statement of [18] and [32], which indicates that there is no way to recover the performance of the permanent magnet if it has occured once. Therefore, it is necessary to protect the demagnetization which occurs in the conventional PMLG although a few research papers are focused on this issue. Extensive research studies indicate that all of the PMLGs used for electrical power generation from the oceanic wave are with fixed length of air gap (FLAG). Almost all of the PMLG has FLAG between its stator and translator throughout the stroke length.

To overcome demagnetization, a new translator design of the PMLG is proposed in this paper by introducing a variable length of air gap (VLAG) topology. The effects of change in the air gap length, velocity and loads are described in a number of research papers [30]. It is analyzed that, if the length of air gap becomes the function of translator velocity, the problem of demagnetization can be eleminated. Therefore, the degradation in electrical power generation can be prevented and the proposed design of the PMLG would be able to generate more electrical power than the conventional PMLG with FLAG topology. Simulation results have shown excellent outcomes of the proposed PMLG design.

\section{Mathematical Model of the Proposed Method}

As the waveform of oceanic wave is close to sine wave and the float with translator follows the wave surface as shown in Fig. 1, the vertical position of translator, $z_{t r}(t)$ and velocity, $v_{t r}(t)$ can be expressed as:

$$
\begin{gathered}
z_{t r}(t)=\frac{H_{t r}}{2} \sin \left(\frac{2 \pi}{T} t \pm \alpha_{i}\right) \\
v_{t r}(t)=\frac{H_{t r} \pi}{T} \cos \left(\frac{2 \pi}{T} t \pm \beta_{i}\right)
\end{gathered}
$$

where $T$ and $H_{t r}$ represent the period and amplitude of the translator motion, respectively. The initial phase angles are symbolized by $\alpha_{i}$ and $\beta_{i}$.

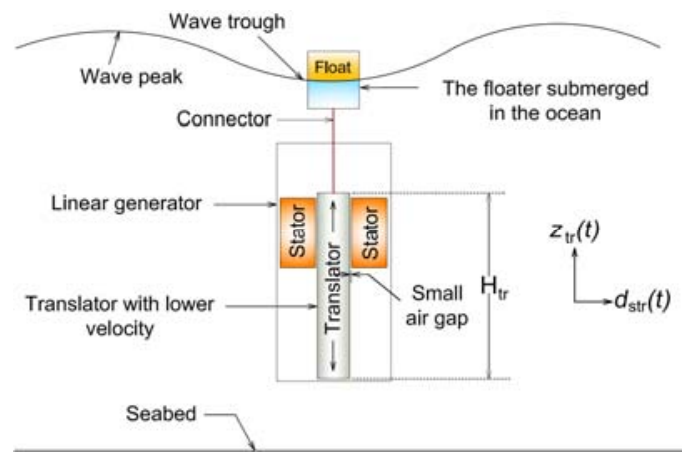

(a)

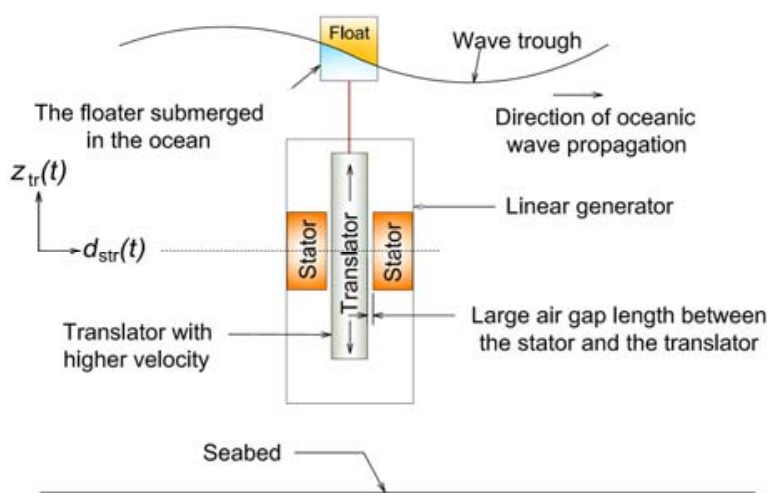

(b)

Fig. 2. Variation of the length of air gap for two different positions. (a) Small air gap length is maintained at the end position of translator. (b) Large air gap is maintained at the vertical center position of translator. 
When the float or buoy lies on the surface of the peak or trough of the oceanic wave, the connected translator reaches at its end position with zero velocity. In other word, the vertical velocity of the translator is zero at the lower end as shown in Fig. 2(a) and also at the upper end. The translator reaches the maximum velocity at its vertical center position as shown in Fig. 2(b) according to (2). For the same length of air gap, the higher velocity of translator would create higher opposing reverse magnetic field against permanent magnets. To minimize this effect, the air gap length, $d_{s t r}(t)$, is represented as a function of position as follows:

$$
d_{s t r}(t)=d_{\min }+d_{\mathrm{var}}\left[1-\frac{\cos \pi\left\{z_{t r}(t)\right\}}{H_{t r}}\right]
$$

where $d_{\min }$ and $d_{v a r}$ represent the minimum and variable air gap lengths, respectively. The flux variation, $\Phi_{s t}$ can be represented as:

$$
\begin{gathered}
\Phi_{s t}(t)=\bar{\Phi} \sin \left(\frac{2 \pi}{\lambda} z_{t r}(t) \pm \alpha_{i}\right)\left[2 f\left\{z_{t r}(t)\right\}-f^{2}\left\{z_{t r}(t)\right]\right. \\
f\left\{z_{t r}(t)\right\}=\frac{\cos \pi\left\{z_{t r}(t)\right\}}{H_{t r}}
\end{gathered}
$$

where $\lambda$ is the wavelength of the oceanic wave. The generated voltage of the proposed linear generator, $V_{\text {gen }}$ can be expressed in the following

$$
\begin{gathered}
v_{\text {gen }}(t)=V_{\max } \cos \left(\frac{2 \pi}{T} t\right) \cos \left\{\frac{2 \pi A_{t r}}{\lambda} \sin \left(\frac{2 \pi}{T} t\right)\right\} \\
{\left[2 f\left\{z_{t r}(t)\right\}-f^{2}\left\{z_{t r}(t)\right\}\right]}
\end{gathered}
$$

The length of air gap increases with the increase of velocity of the translator and vice-versa. The vertical displacement and velocity of the translator are related to the float, which can be realized from Fig. 3 .

\section{MinimiZation of DemagnetiZation}

Fig. 3 shows the nature of vertical position and velocity of a translator with respect to time in all PMLGs. The generated voltage, electrical power, and hence the armature current are directly proportional to the translator velocity considering the same load where the nature of current and power of the PMLG are shown in Fig. 3. The characteristic curve of any permanent magnet is generally represented by $B-H$ loop where $B$ is the magnetic flux density and $H$ the magnetic field intensity. During the operation of electrical power generation, by Lenz's law, these permanent magnets face the opposite magnetic field intensity from the armature as shown in Fig. 4. As a result, demagnetization takes place on a previously magnetized hard magnetic material which is not been used in an electrical power generator.

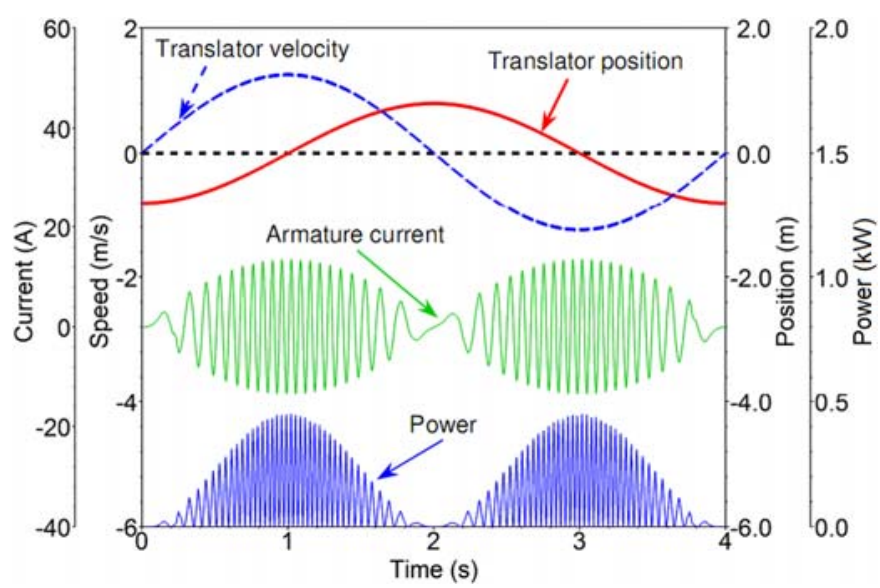

Fig. 3. The winding current and power generation of a PMLG with respect to the translator position and velocity.

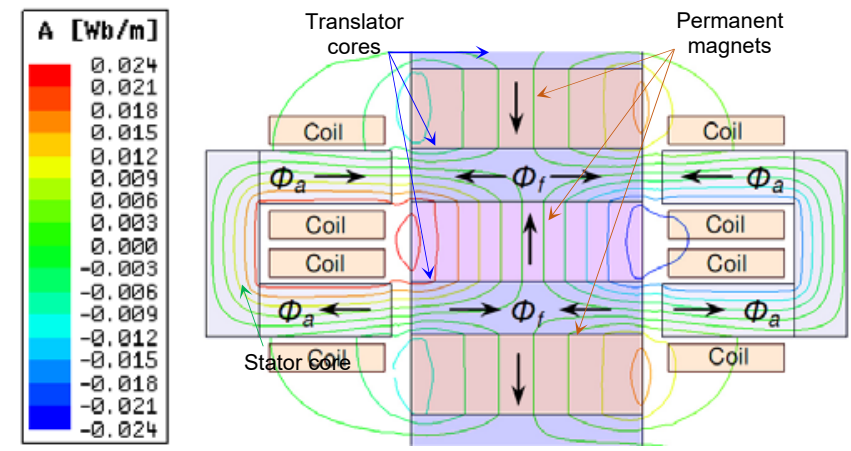

Fig. 4. Demagnetization occurs in permanent magnet of the existing PMLG.

For this reason, the second quadrant of the characteristics curve is considered for analysis as shown in Fig. 5 where the remanent magnetism is denoted by $B_{r}$ and the coercive force by $H_{c}$. Before starting operation as a generator, the magnetic remanence, $B_{r}$ is located at point $a$ according to Fig. 5. The induced emf, $E_{p}$ per phase can be expressed as

$$
E_{p}=\pi \sqrt{2} f N_{1} k_{w l} \varphi_{g}
$$

where the winding factor is denoted by $k_{w l}$ and $f$ is the frequency of generated voltage. The magnetic flux in the air gap, $\Phi_{g}$ can be represented as

$$
\varphi_{g}=\varphi_{f}-\varphi_{a}-\varphi_{l}
$$

where $\Phi_{f}$ is the excitation flux, $\Phi_{a}$ the flux created from armature reaction, and $\Phi_{l}$ the leakage flux as shown in Fig. 4. As the value of $\Phi_{l}$ is very small compared to $\Phi_{f}$ and $\Phi_{a}$, under no load condition (without armature reaction), $\Phi_{g}$ is nearly equal to $\Phi_{f}$. For the armature reaction, $\Phi_{a}$ mainly opposes the excitation flux which occurs during the operation of electrical power generator. 


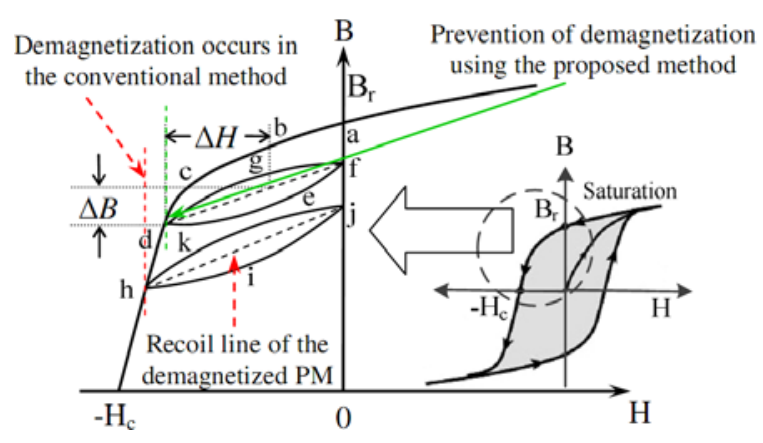

Fig. 5. Demagnetization curve of a permanent magnet (PM).

In Fig. 5, points $a, b, c, d$, and $h$ lie on the demagnetization curve where $a$ represents $B_{r}$ of a newly installed permanent magnet. Because of the reverse magnetic field, $\Phi_{a}$ in the opposite direction of excitation field, $B$ of the permanent magnet falls down to for example point $d$ from point $a$ followed by the abcd line. In the stationary state or under no load condition, $B$ reaches point $f$ followed by the def line instead of returning to point $a$ as before. As a result, the value of $B_{r}$ drops down to point $f$ from point $a$. Re-applying the same amount of $-H$ would again cause the same lower value of $B_{r}$ from point $f$ to $d$ followed by $f g d$. Under no load condition, the value of $B$ again reaches point $f$ followed by def again making a secondary hysteresis loop with a recoil line $d f$. This degraded value of $B_{r}$ which falls to point $f$ will never reach point $a$. The recoil permeability, $\mu_{\text {rec }}$ can be expressed by slope of $d f$ line which is considered for simplification.

For the lower value of applied $-H$, the magnetic flux density would not further decrease more than point $d$. On the other hand, for the higher value of applied $-H$, the magnetic flux density would decrease from point $d$ to for example point $h$ which would never reach point $f$. Another secondary loop, $h i j k h$ is made with a recoil line $h j$ and the value of $B_{r}$ would never reach any higher value than point $f$. This phenomenon happens to the conventional FLAG PMLG automatically because of the variable translator velocity created by natural oceanic wave. As the magnitude of reverse magnetic field depends on the velocity of the PMLG for a particular load which varies with wave motion, $B$ would be reduced to a new value.

\section{Design OF THE PRoposed PMLG}

Fig. 6(a) illustrates the construction of the proposed VLAG PMLG where the magnetic cores and permanent magnets are labeled. Fig. 6(b) represents the equivalent circuit diagram of the winding. In Fig. 6(b), $E_{C-1}, E_{C-2}, E_{C-3}$, and $E_{C-4}$ represent the coil voltages of Coil-1, Coil-2, Coil-3, and Coil-4, respectively as labeled in Fig. 6(a). Copper wires are selected for coil windings. Two stators with windings are located on the left and the right sides of the translator.

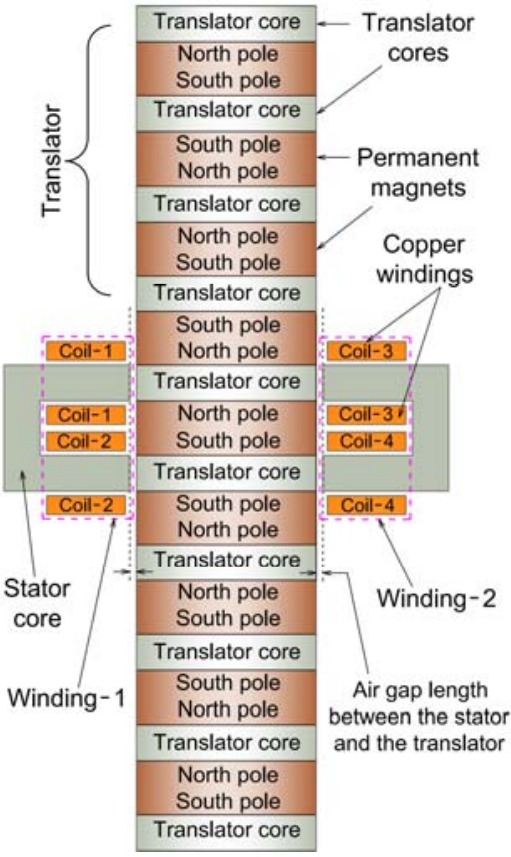

(a)

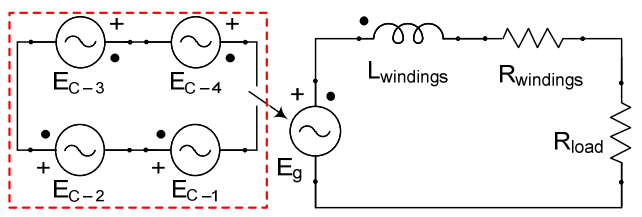

(b)

Fig. 6. (a) Construction of the PMLG and (b) its equivalent circuit diagram.

Each of the stator winding consists of two coils which are opposite in electrical phase from each other. Fig. 6(b) shows the equivalent circuit diagram of the proposed VLAG PMLG where four coils are connected in series. The equivalent series resistance is denoted by $R_{\text {windings }}$ and the equivalent series inductance is denoted by $L_{\text {windings }}$.

The total voltage, $E_{g}$ generated from these four coils are marked by a dashed area. The translator is allowed to move vertically between the stators. The alignment of the magnetic poles and direction of flux lines can also be understood from Fig. 6 (a). Fig. 7 represents the translator pole pitch, $\tau_{t r}$, which represents the height of two consecutive translator poles with permanent magnets. Three phase voltages are produced due to the stator phase difference which is also shown in Fig. 7. The vertical distance between the stator producing phase-a and phase-c, $H_{\Phi a-\Phi c}$ can be represented in the following

$$
H_{\Phi a-\Phi c}=2\left(\tau_{t r}+\frac{1}{3} \tau_{t r}\right)
$$




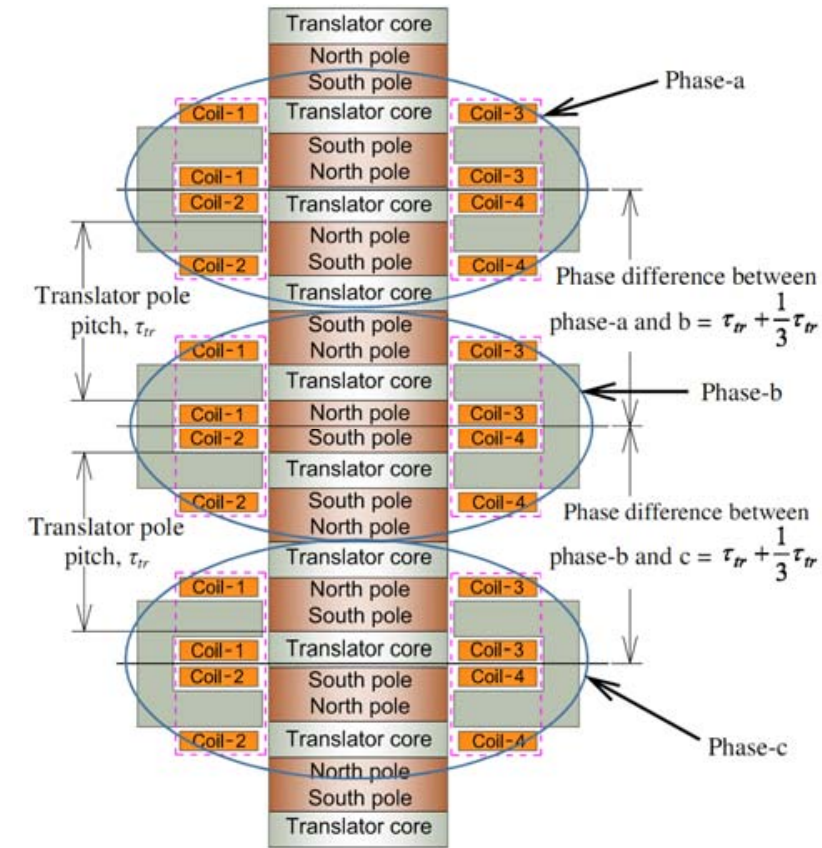

Fig. 7. Stator arrangement of the proposed PMLG.

Fig. 8 illustrates the construction of the translator of the proposed PMLG which consists of some steel cores and permanent magnets. The variation of air gap length, $\Delta d_{s t r}$ is very small compared to the length of the translator. For this reason, the translator is exaggerated so that the curvature can be seen clearly. The number of steel cores are represented by $n$, where the maximum value of $n, n_{\max }=24$. Considering the steel core placed in the vertical centre which has $n=0$, the total number of steel cores in the translator is $2 \times n_{\max }+1$. On the other hand, the numbers of permanent magnets are denoted by $m$. Since two opposite orientations of permanent magnets are used to design the translator, they are represented by $\mathrm{PM}(+\mathrm{z})_{n}$ and $\operatorname{PM}(-\mathrm{z})_{n}$. The permanent magnets in which the North poles are placed on the upper side and the South poles in the lower side, are denoted as $\mathrm{PM}(+\mathrm{z})_{n}$. Similarly, $\mathrm{PM}(-\mathrm{z})_{n}$ represents the same with opposite orientation. The total number of $\mathrm{PM}(+\mathrm{z})_{n}$ and $\mathrm{PM}(-\mathrm{z})_{n}$ in the translator is $2 \times m_{\max }$ where $m_{\max }=12$. The distance between the stator and translator core is denoted as $\Delta_{\mathrm{SC}-n} d_{s t r}$, where $-24 \leq n \leq 24$. Similarly, the distance between the stator permanent magnet is denoted as $\Delta$ PM- $m d_{s t r}$ where $-12 \leq m \leq 12$ and $m \neq 0$. For the consecutive steel cores and permanent magnets the value of $\Delta_{\mathrm{SC}-n} d_{s t r}$ slightly differs from that of the $\Delta_{\mathrm{PM}-m} d_{s t r}$. The value of $\Delta_{\mathrm{SC}-n} d_{s t r}$ and $\Delta_{\mathrm{PM}-m} d_{s t r}$ is higher at both ends and lower at the vertical centre of the translator. The slope of $n$-th translator core, $M_{S C-n}$, and $m$-th permanent magnet, $M_{P M-m}$ can be represented as

$$
M_{s c-n}= \pm \frac{Z_{S C-H}}{\Delta_{P M-m} d_{s t r}}, M_{P M-m}= \pm \frac{Z_{P M-H}}{\Delta_{P M-m} d_{s t r}}
$$

where $Z_{S C-H}$ and $Z_{P M-H}$ are the thickness (height) of the translator core and the permanent magnet, respectively. Only for $n=0$, the slope of the translator core is infinite, because its left and right sides are vertical.

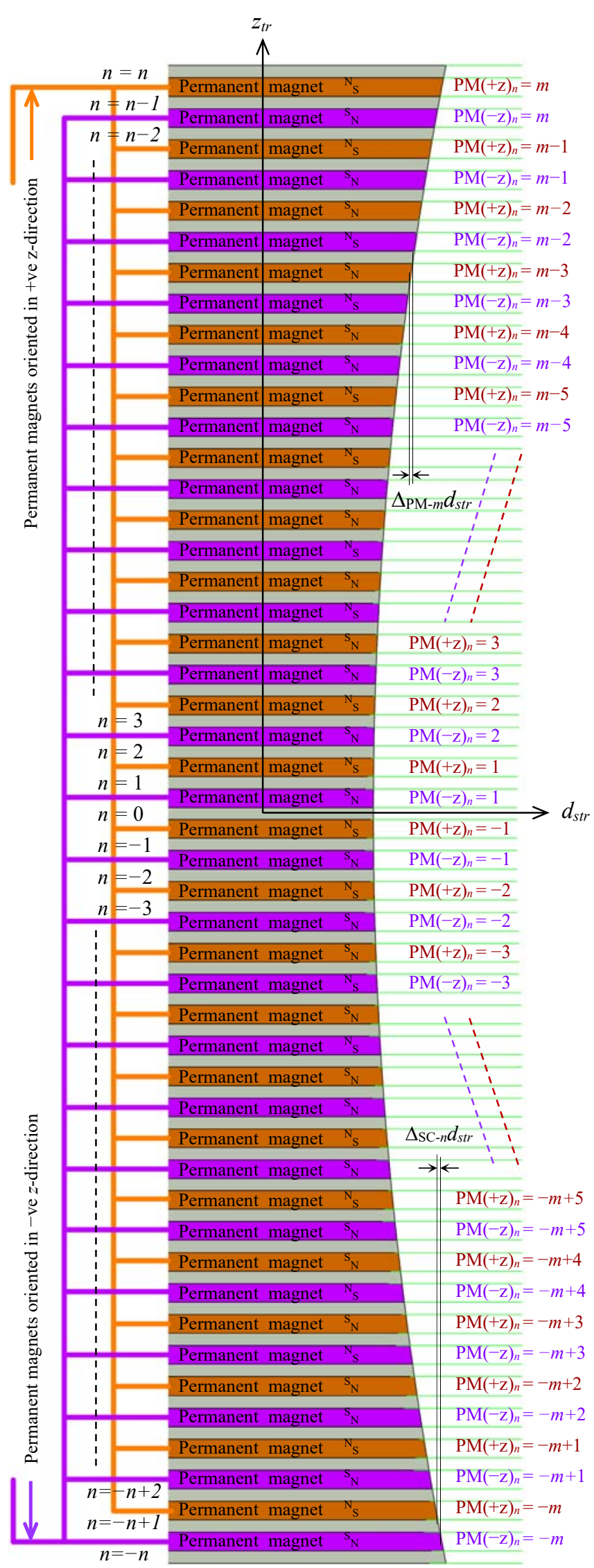

Fig. 8. Construction of the translator of the proposed PMLG. 


\section{Simulation Results}

In the simulation setup, the translator is allowed to move vertically. The peak to peak vertical travelling distance of the translator is $1.6 \mathrm{~m}$ which is known as stroke length. The time period of the oceanic wave is set to $4 \mathrm{~s}$. The velocity of the translator ranges from $0-1.25 \mathrm{~m} / \mathrm{s}$ depending on time. As the nature of the oceanic wave is almost sinusoidal, in the motion setup, the time period of sine wave motion is $4 \mathrm{~s}$. The width and depth of a single unit of the proposed PMLG are $0.196 \mathrm{~m}$ and $0.1 \mathrm{~m}$, respectively. The induced voltages and currents of phase-a, phase- $b$, and phase-c of the proposed PMLG are plotted in Figs. 9 and 10, respectively, for the resistive load of $4 \Omega$. The curved lines represent the amplitude of the load currents for the same generator with the fixed length of air gaps as in the conventional linear genetator.

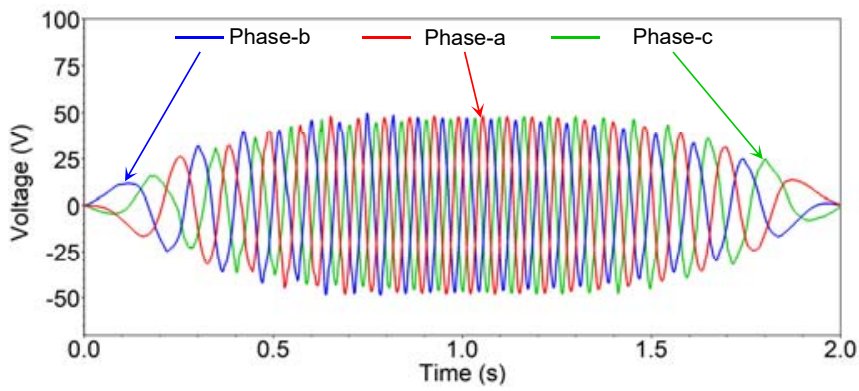

Fig. 9. The induced voltages of the proposed linear generator.

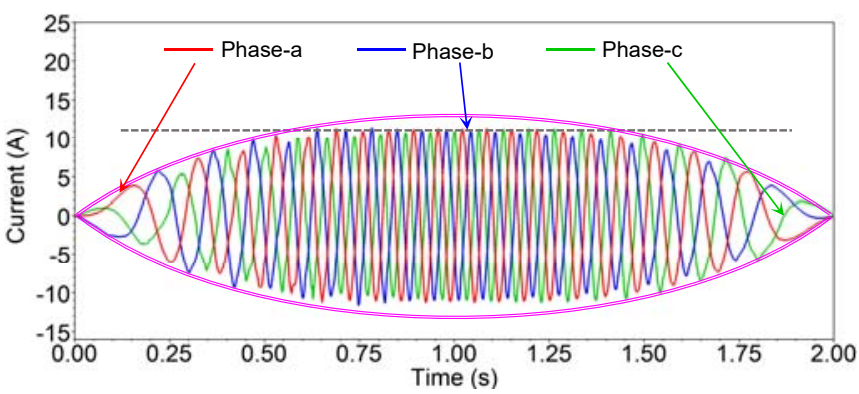

Fig. 10. The load currents of the proposed linear generator.

The proposed linear generator can limit the armature current to a specified maximum value inherently to prevent demagnetization as shown in Fig. 11 for phase-a. When the current reaches its limit, the amplitude becomes constant which is represented by the straight lines.

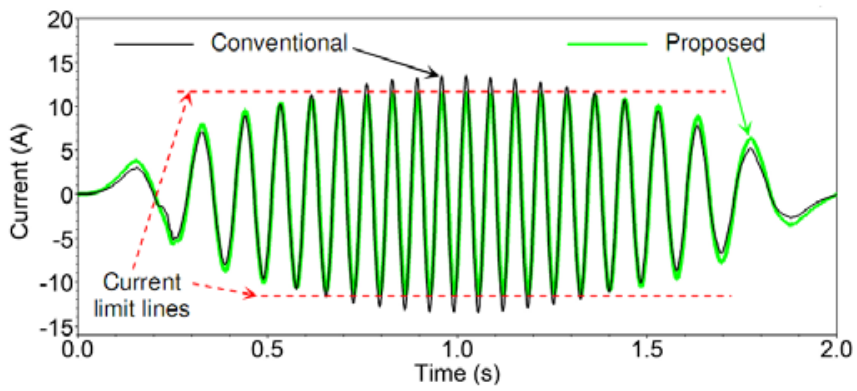

Fig. 11. The armature current of the conventional FLAG and the proposed VLAG PMLG for a specific load.
The stator winding current waveforms are simulated for the conventional fixed air gap PMLG and the proposed adjustable air gap PMLG, respectively, considering the same design and parameters. The induced voltage and load voltage of phase-a are plotted in Fig. 12. In phase-a, the rms value of load voltage is $24.88 \mathrm{~V}$ which is less than the generated voltage $26.74 \mathrm{~V}$ because of the armature resistance. Similar results are obtained in phase-b and phase-c because of their same armature resistances.

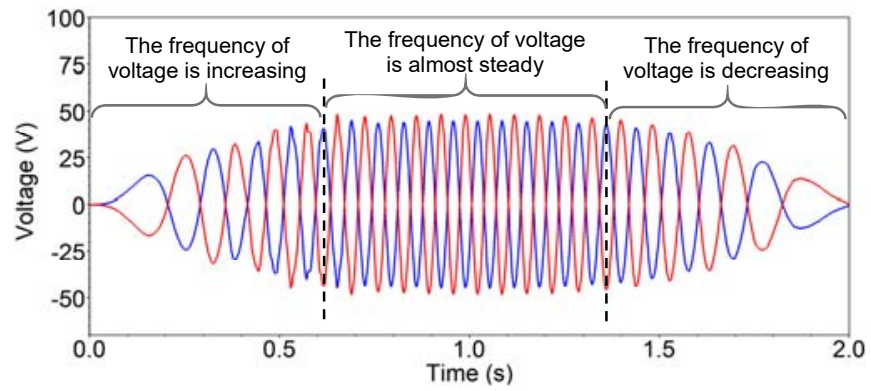

Fig. 12. The induced and load voltage of the proposed linear generator.

The eddy current, hysteresis, and total core losses are shown in Fig. 13, where the core loss components are zero at time, $t=0 \mathrm{~s}, 2 \mathrm{~s}$, and $4 \mathrm{~s}$. The reason is that the relative velocity of the translator with respect to the stator is zero at time $t=0 \mathrm{~s}$, $2 \mathrm{~s}$, and $4 \mathrm{~s}$ which can be realized from Fig. 3. As a result, the generated voltage, current, and frequency are zero at these times and for this reason the core loss is also zero. The core loss gradually increases with the increase of the generated frequency as shown in Figs. 12 and 13 by "Increasing frequency". However, the amount of core loss is almost constant from $t=0.65 \mathrm{~s}-1.35 \mathrm{~s}$ (in the first half cycle) and $t=2.65 \mathrm{~s}-3.35 \mathrm{~s}$ (in the second half cycle) because in these time intervals the frequency is almost constant which is shown in Figs. 12 and 13 by "Similar frequency," which means that the frequency is not exactly the same but the variation of frequency is low. In the same way, the amount of core loss gradually decreases from $1.35 \mathrm{~s}$ in the first half cycle. The applied and generated mechanical forces of the translator are shown in Fig. 14 for one cycle of the oceanic wave.

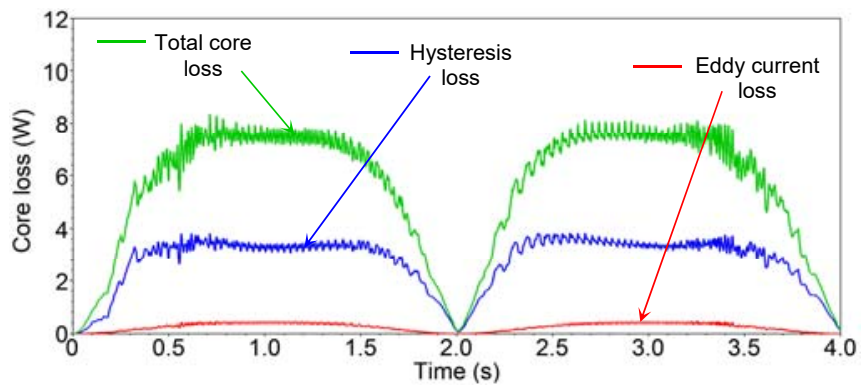

Fig. 13. The core loss profile of the proposed linear generator.

The value of the applied force, $F_{a z}$ is negative in the first half cycle because the translator moves in the upward direction which can be understood from Fig. 3. Now, if the direction of force is considerd downward, the value of $F_{a z}$ in the first half cycle is negative. Similarly, the value of $F_{a z}$ is 
positive in the second half cycle because of the translator's downward motion. The generated force, $F_{g x}$ shown in Fig. 14 is due to the electromagnetic interaction between the translator and stator.

The generated electrical powers for different loads are shown in Fig. 15 and tabulated in Table I for comparison. The generated powers for $4 \Omega, 6 \Omega$, and $8 \Omega$ loads are denoted as $\mathrm{P}_{4 \Omega}, \mathrm{P}_{6 \Omega}$, and $\mathrm{P}_{8 \Omega}$, respectively. The terminal voltage, load current, and magnetic flux linkage are shown in Fig. 16, where the magnetic flux linkage is more at the two ends and low at the centre of the half cycle.

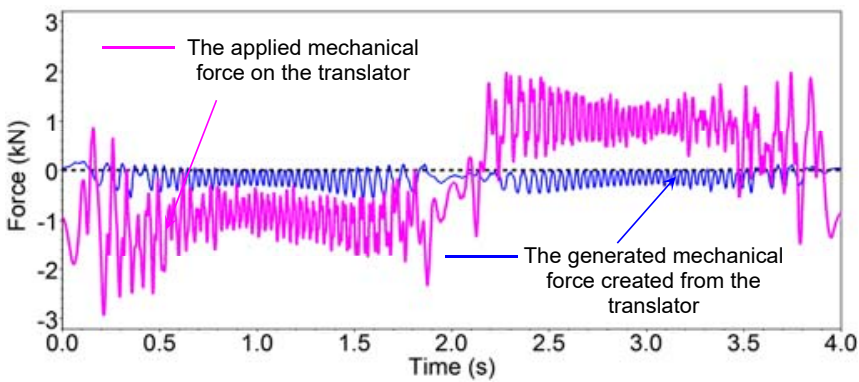

Fig. 14. The applied and generated forces of the translator of the proposed linear generator.

TABLE I

DIMENSIONS OF THE PMLG

\begin{tabular}{|c|c|c|c|}
\hline Power & Average value $(\mathrm{W})$ & Peak value $(\mathrm{W})$ & Rms value $(\mathrm{W})$ \\
\hline $\mathrm{P}_{4 \Omega}$ & 163.336 & 519.733 & 160.009 \\
\hline $\mathrm{P}_{6 \Omega}$ & 116.155 & 374.675 & 115.393 \\
\hline $\mathrm{P}_{8 \Omega}$ & 89.100 & 291.372 & 88.584 \\
\hline
\end{tabular}

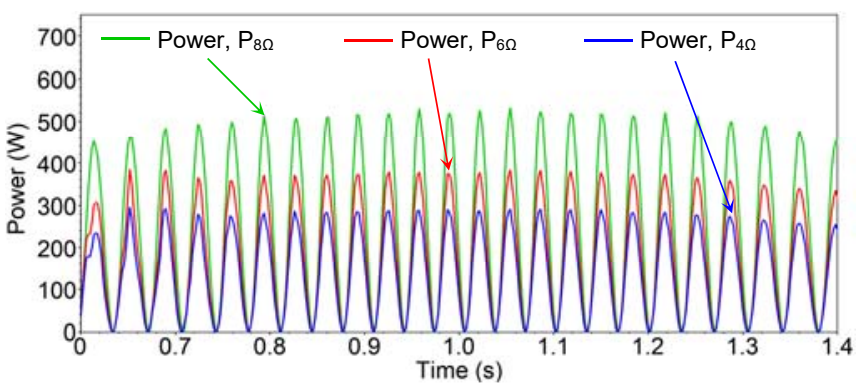

Fig. 15. The generated powers for different loads.

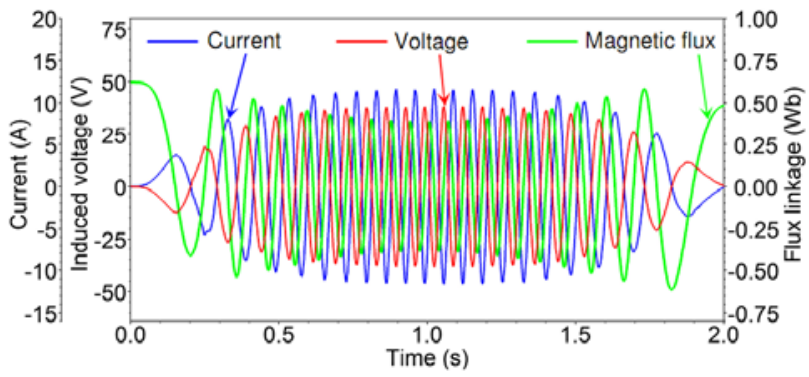

Fig. 16. Voltage, current, and magnetic flux of the VLAG PMLG.

It indicates that the proposed linear generator protect overcurrent flowing through the coil by minimizing flux linkage. Fig. 17 presents the simulated armature current waveform for three loads, viz. $I_{1}(8 \mathrm{~A}), I_{2}(10 \mathrm{~A})$, and $I_{3}(12$ A). Fig. 18 plots the waveform of electrical power generation for the same load maintaining the same maximum current rating for the FLAG and VLAG PMLG to make a comparison.

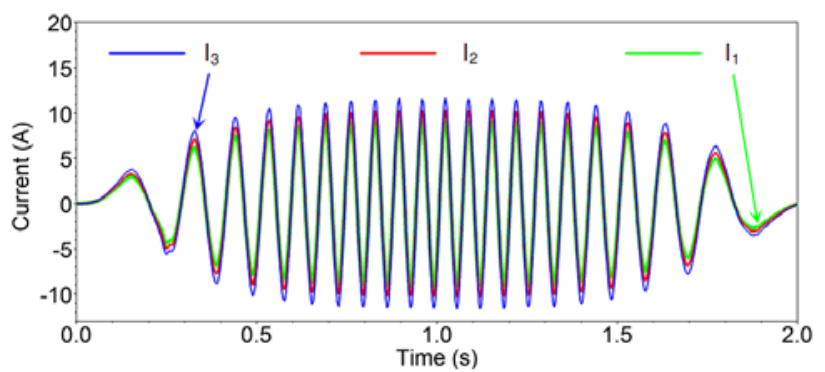

Fig. 17. Different armature currents of the VLAG PMLG.

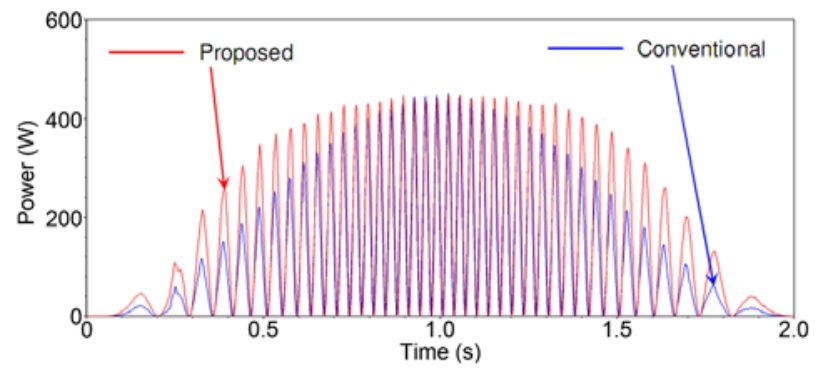

Fig. 18. Generated electrical power for FLAG and VLAG PMLG.

It can be seen form Fig. 18 that, although the peak value of power is the same, the average electrical power of the proposed linear generator is larger compared to that of the conventional one. Figs. 19-22 show the magnetic properties of the proposed VLAG PMLG. The magnetic flux density of VLAG PMLG is shown in Fig. 19 for the maximum velocity of the translator at the moment of alignment with stator cores. The length of air gap needs to maintain a heigher value, not to allow the coil current beyond the specified limit.

The magnetic flux density of the same PMLG is shown in Fig. 20 for the minimum value of translator speed at the moment of alignment with stator cores. It happens at the end positions of the translator. The length of air gap needs to maintain a lower value in this case so that the magnetic flux linkage can be reached to higher value to boost up the coil current, also maintaining the current limit. Different parameters of the PMLG are tabulated in Table II. Figs. 21 and 22 present the magnetic field intensity, $H$ of the proposed VLAG PMLG for maximum and minimum lengths of the air gap at the highest and lowest translator velocities, respectively, at the moment of vertical alignments of the stator and translator cores.

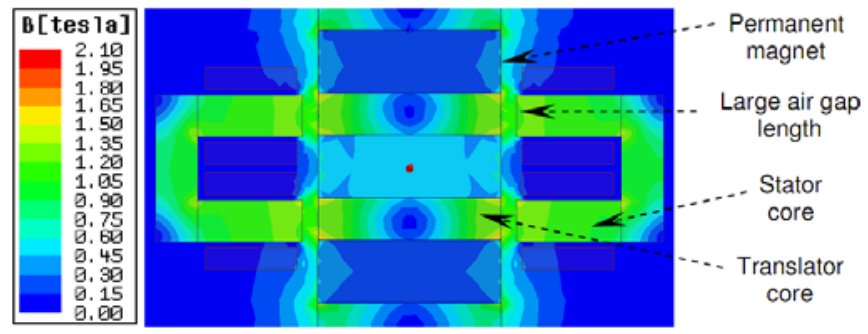

Fig. 19. Magnetic flux density of the VLAG PMLG for maximum air gap. 


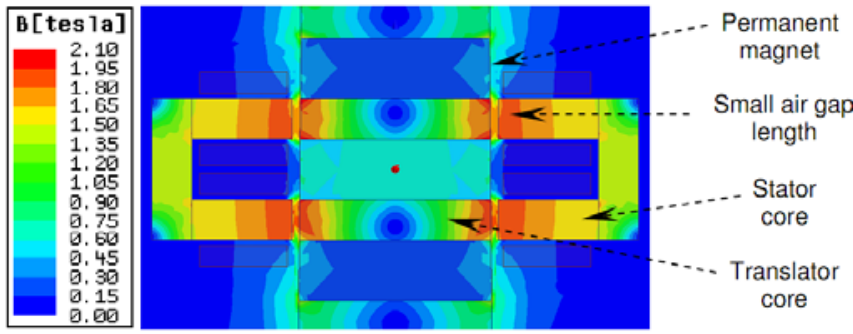

Fig. 20. Magnetic flux density of the VLAG PMLG for minimum air gap.

TABLE II

DIMENSIONS OF THE PMLG

\begin{tabular}{|l|c|}
\hline Name of the items & Value \\
\hline Air gap length between the stator and translator $(\mathrm{mm})$ & $3 \sim 6$ \\
\hline Thickness of the stator pole $(\mathrm{cm})$ & 1.6 \\
\hline Width of the stator pole shoe $(\mathrm{cm})$ & 4 \\
\hline Thickness of the permanent magnet $(\mathrm{cm})$ & 2.4 \\
\hline Vertical distance of the stator poles $(\mathrm{cm})$ & 4 \\
\hline Thickness of the translator core $(\mathrm{cm})$ & 1.6 \\
\hline Vertical distance of the translator for one cycle $(\mathrm{cm})$ & 8 \\
\hline Height of the stator pole shoe $(\mathrm{cm})$ & 1.6 \\
\hline Length of the cross section of the copper coil $(\mathrm{cm})$ & 3.5 \\
\hline Width of the cross section of the copper coil $(\mathrm{mm})$ & 8.5 \\
\hline Total width of the stator poles $(\mathrm{cm})$ & 5.6 \\
\hline
\end{tabular}
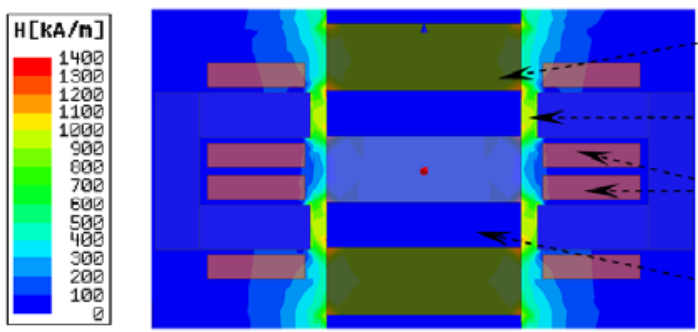

Permanent magnet

$H$ in large air gap length

$==-$ Stator coils

Translator core

Fig. 21. Magnetic field intensity of the VLAG PMLG for maximum air gap.
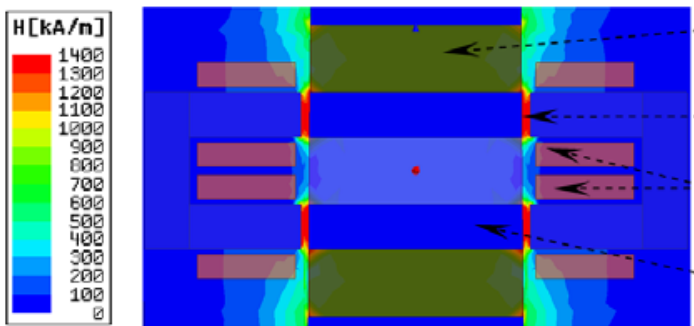

- Permanent
magnet

$H$ in low air gap length

Stator coils

Translator core

Fig. 22. Magnetic field intensity of the VLAG PMLG for minimum air gap

Fig. 23 represents the simulated and experimental load voltages of the PMLG with the conventional fixed length of air gap. As the load is resistive, the nature of current waveform and voltage waveform is the same. The magnitude of generated voltage exceeded the limit for which excess amount of armature current would occur. Fig. 24 represents the simulated and experimental load voltages of the PMLG with the proposed variable length of air gap. The magnitude of generated voltage did not exceed the limit which ensures that, excess amount of armature current would not occur. In the experimental result, the proposed VLAG PMLG vibrates for the lower linear velocities at $t=0 \mathrm{~s}$ and $t=2 \mathrm{~s}$. As a result, the generated voltage fluctuates at these moments which can be seen at Figs. 23 and 24.

The downscale prototype of the proposed PMLG connected to a resistive load with the translator design is shown in Fig. 25. The translator with the proposed VLAG PMLG is mounted in a linear motion generator as shown in Fig. 25 (a).

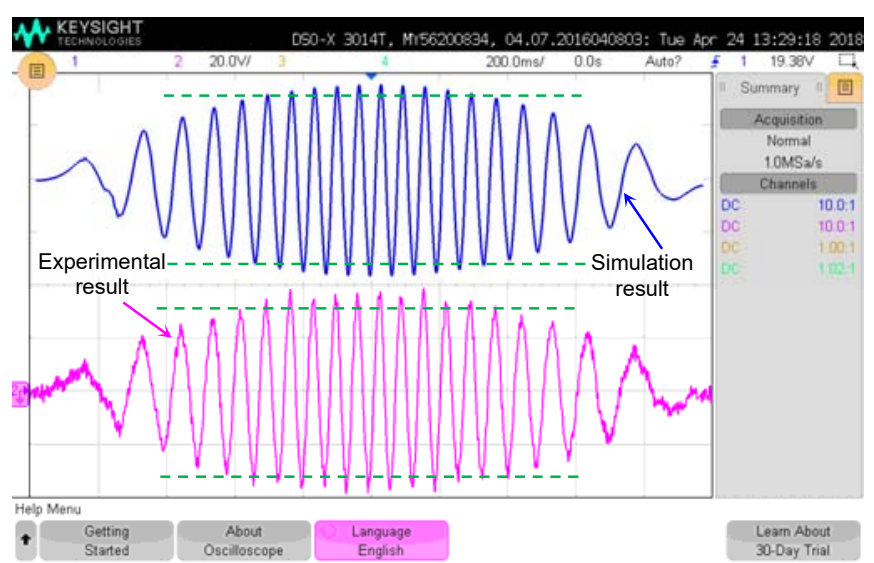

Fig. 23. The load voltage of the conventional linear generator.

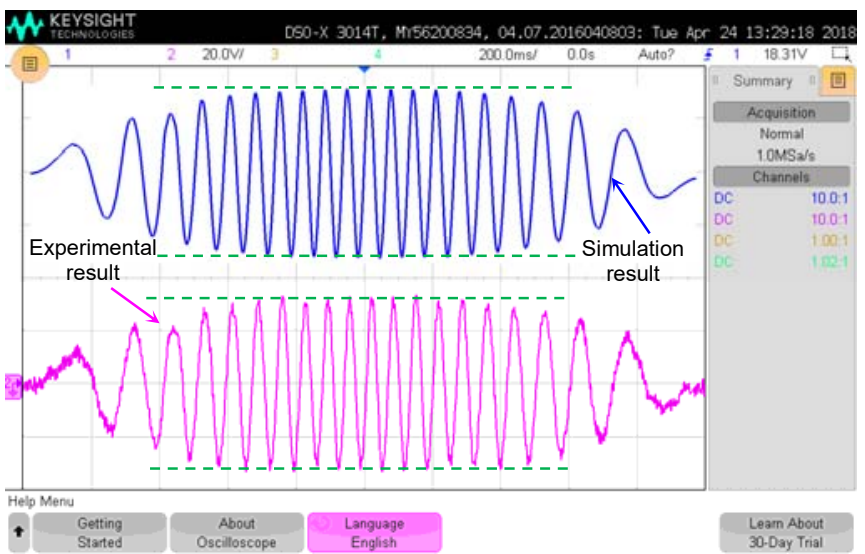

Fig. 24. The load voltage of the proposed linear generator.

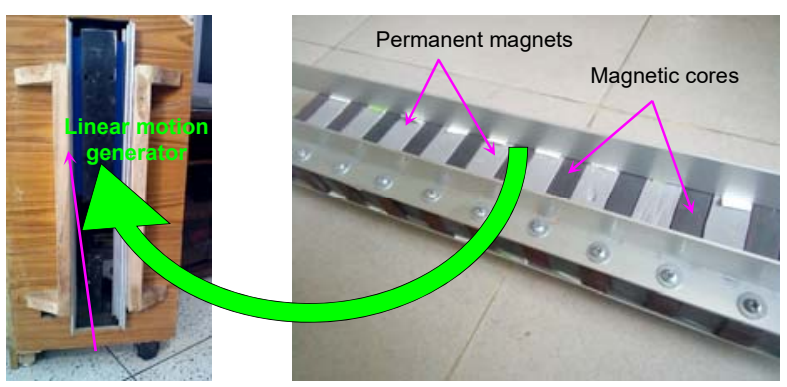

(a)

(b)

Fig. 25. (a) Linear motion generator and (b) translator design with variable air gap topology.

To produce high amount of electrical power, the depth of a single unit of the proposed PMLG is increased from $0.1 \mathrm{~m}$ to 1 $\mathrm{m}$ in the simulation setup. The peak generated power of the single unit is then increased from around $450 \mathrm{~W}$ to $5 \mathrm{~kW}$. Four units are cascaded together to generate $20 \mathrm{~kW}$ electrical power as shown in Fig. 26. Four translators are mounted together at their top and bottom side to ensure free vertical movement of them. The width, depth and height of the cascaded VLAG PMLG are $1 \mathrm{~m}, 1 \mathrm{~m}$, and $0.35 \mathrm{~m}$ respectively. The output 
power can be increased to an even higher value by adding more units together.

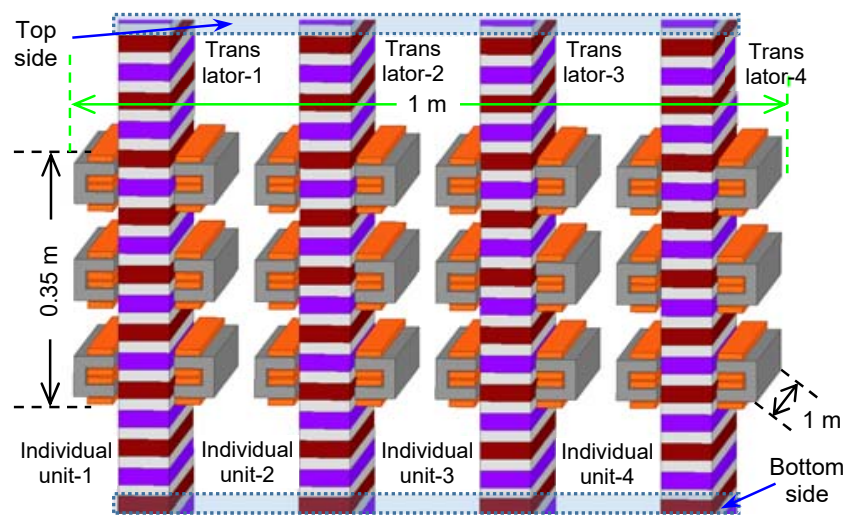

Fig. 26. Cutaway drawing of the proposed cascaded VLAG PMLG.

\section{CONCLUSION}

A new design is proposed to various PMLGs to avoid demagnetization. A downscale prototype is fabricated to test the performance of the proposed VLAG PMLG. The numerical simulation and experimental test results are very close to each other, and both demonstrate that the proposed PMLG can effectively solve the problem of the demagnetization of permanent magnets. The VLAG PMLG can generate more average electrical power under the same operating condition, which is another benefit. For a particular load and translator speed as shown in the paper, the VLAG PMLG can generated $23.77 \%$ more electrical power than the FLAG PMLG for the same operating point on the curve of demagnetization and armature current limit. Due to the prevention of demagnetization, degradation of power generation can be prevented. The proposed cascaded VLAG PMLG can generate $20 \mathrm{~kW}$ electrical power.

\section{REFERENCES}

[1] P. Rodriguez, "Equivalent model of large-scale synchronous photovoltaic power plants," IEEE Trans. Ind. Appl., vol. 52, no. 6, pp. 5029-5040, 2016

[2] S. Zhu, Y. Zhang, and A. A. Chowdhury, "Capacity credit of wind generation based on minimum resource adequacy procurement," IEEE Trans. Ind. Appl., vol. 48, no. 2, pp. 730-735, 2012.

[3] Y. Hu, S. Member, C. Gan, W. Cao, S. Member, Y. Fang, S. J. Finney, and J. Wu, "Solar PV-powered SRM drive for EVS with flexible energy control functions," IEEE Trans. Ind. Appl., vol. 52, no. 4, pp. $3357-$ 3366, 2016.

[4] H. M. Al-masri, S. Member, M. Ehsani, and L. Fellow, "Feasibility investigation of a hybrid on-grid wind photovoltaic retrofitting system," IEEE Trans. Ind. Appl., vol. 52, no. 3, pp. 1979-1988, 2016.

[5] A. Reznik, M. G. Simões, S. Member, A. Al-durra, S. M. Muyeen, S Member, A. P. E. Modeling, and L. C. L. Filter, "LCL filter design and performance analysis for grid-interconnected systems," IEEE Trans. Ind. Appl., vol. 50, no. 2, pp. 1225-1232, 2014

[6] B. Ke, T. Ku, Y. Ke, and S. Member, "Sizing the battery energy storage system on a university campus with prediction of load and photovoltaic generation," IEEE Trans. Ind. Appl., vol. 52, no. 2, pp. 1136-1147, 2016.

[7] R. C. Dugan, J. A. Taylor, and D. Montenegro, "Energy storage modeling for distribution planning," IEEE Trans. Ind. Appl., vol. 53, no 2, pp. 954-962, 2017.

[8] A. R. A. Arkadan, N. Al-Aawar, and A. O. Hariri, "EM-Taguchi module for characterization of WAD," IEEE Trans. Magn., vol. 51, no. 3, article 8202304, Mar. 2015.
[9] A. Clément, et al., "Wave energy in Europe: Current status and perspectives," Renew. Sustain. Energy Rev., vol. 6, no. 5, pp. 405-431, Oct. 2002.

[10] H. Polinder, J. G. Slootweg, M. J. Hoeijmakers, and J. C. Compter, "Modeling of a linear PM machine including magnetic saturation and end effects: maximum force-to-current ratio," IEEE Trans. Ind. Appl., vol. 39 pp. 1681-1688, 2003

[11] N. Bianchi and S. Bolognani, "Design techniques for reducing the cogging torque in surface-mounted PM motors," IEEE Trans. Ind. Appl., vol. 38 , no. 5, pp. 1259-1265, 2002.

[12] L. Dosiek and P. Pillay, "Cogging torque reduction in permanent magnet machines," IEEE Trans. Ind. Appl., vol. 43, no. 6, pp. 1565-1571, Nov./Dec. 2007.

[13] J. X. Jin, L. H. Zheng, Y. G. Guo, and J. G. Zhu, "Performance characteristics of an HTS linear synchronous motor with HTS bulk magnet secondary," IEEE Trans. Ind. Appl., vol. 47, no. 6, pp. 24692477, Nov./Dec.2011.

[14] J. Prudell, M. Stoddard, E. Amon, T. K. A. Brekken, and A. V. Jouanne, "A permanent-magnet tubular linear generator for ocean wave energy conversion," IEEE Trans. Ind. Appl., vol. 46, no. 6, pp. 2392-2400, Nov./Dec. 2010.

[15] L. Cappelli, F. Marignetti, G. Mattiazzo, E. Giorcelli, G. Bracco, S. Carbone, and C. Attaianese, "Linear tubular permanent-magnet generators for the inertial sea wave energy converter," IEEE Trans. Ind. Appl., vol. 50, no. 3, pp. 1817-1828, May/Jun. 2014.

[16] R. Vermaak and M. J. Kamper, "Experimental evaluation and predictive control of an air-cored linear generator for direct-drive wave energy converters," IEEE Trans. Ind. Appl., vol. 48, no. 6, pp. 1817-1826, Nov./Dec. 2012.

[17] S. T. Boroujeni, J. Milimonfared, and M. Ashabani, "Design, prototyping, and analysis of a novel tubular permanent-magnet linear machine," IEEE Trans. Magn., vol. 45, no. 12, pp. 5405-5413, Dec. 2009.

[18] L. Huang, H. Yu, M. Hu, J. Zhao, and Z. Cheng, "A novel flux-switching permanent-magnet linear generator for wave energy extraction application," IEEE Trans. Magn., vol. 47, no. 5, pp. 1034-1037, May 2011.

[19] J. A. García-Alzórriz, J. Grau, R. Córdoba, and J. Muela, "A novel double-sided flat rectangular linear permanent magnets synchronous generator for sea wave energy application," in Proc. the 7th Int. Conf. Electrical and Electronics Engineering, Bursa, 2011, pp. I-248-252.

[20] O. Farrok and M. M. Ali, "A new technique to improve the linear generator designed for oceanic wave energy conversion," in Proc. Int. Conf. Electrical and Computer Engineering, Dhaka, Bangladesh, Dec. 20-22, 2014, pp. 714-717.

[21] O. Farrok, M. R. Islam, and M. R. I. Sheikh, "Fuzzy logic based an improved controller for wave energy conversion systems," in Proc. Int. Conf. Electrical Engineering and Information Communication Technology, Dhaka, Bangladesh, May 21-23, 2015, pp. 1-6.

[22] G. Bramerdorfer, W. Amrhein, S. M. Winkler, and M. Affenzeller, "Identification of a nonlinear PMSM model using symbolic regression and its application to current optimization scenarios," in Proc. $40^{\text {th }}$ Annu. Conf. IEEE Ind. Electron. Society, Dallas, USA, 2014, pp. 628-633.

[23] O. Farrok, M. R. Islam, M. R. I. Sheikh, and W. Xu, "A new optimization methodology of the linear generator for wave energy conversion systems," in Proc. IEEE Int. Conf. Ind. Technol., Taipei, Taiwan, Mar. 14-17, 2016, pp. 1412-1417.

[24] G. Bramerdorfer, A. C. Zăvoianu, S. Silber, E. Lughofer, and W. Amrhein, "Possibilities for speeding up the FE-based optimization of electrical machines-A case study," IEEE Trans. Ind. Appl., vol. 52, no. 6, pp. 4668-4677, Nov.-Dec. 2016.

[25] O. Farrok, M. R. Islam, M. R. I. Sheikh, Y. Guo, J. Zhu, and G. Lei, "A novel method to avoid degradation due to demagnetization of PM linear generators for oceanic wave energy extraction," in Proc. 20th Int. Conf. Electr. Mach. Syst., Sydney, Australia, 2017, pp. 1-6.

[26] L. Huang, J. Liu, H. Yu, R. Qu, H. Chen, and H. Fang, "Winding configuration and performance investigations of a tubular superconducting flux-switching linear generator," IEEE Trans. Appl. Supercond., vol. 25, no. 3, 2015.

[27] J. Wang et al., "Design of a superconducting synchronous generator with LTS field windings for $12 \mathrm{MW}$ offshore direct-drive wind turbines," IEEE Trans. Ind. Electron., vol. 63, no. 3, pp. 1618-1628, Mar. 2016.

[28] L. J. Ba, J. X. Jin, Z. H. Wu, and L. H. Zheng, "Conceptual design of an HTS wave linear generator," IEEE Trans. Appl. Supercond., vol. 26, no. 7, pp. 1-6, Oct. 2016.

[29] O. Farrok, M. R. Islam, M. R. I. Sheikh, Y. G. Guo, J. G. Zhu, and W. 
$\mathrm{Xu}$, "Analysis and design of a novel linear generator for harvesting oceanic wave energy," in Proc. IEEE Int. Conf. Appl. Supercond. Electromagn. Devices, Shanghai, China, Nov. 20-23, 2015, pp. 272 273.

[30] O. Farrok, M. R. Islam, M. R. I. Sheikh, Y. G. Guo, J. G. Zhu, and W. $\mathrm{Xu}$, A novel superconducting magnet excited linear generator for wave energy conversion system," IEEE Trans. Appl. Supercond., vol. 26, no. 7, art. 6207105, Oct. 2016.

[31] I. Stamenkovic, N. Milivojevic, N. Schofield, and M. Krishnamurthy, "Design, analysis, and optimization of ironless stator permanent magnet machines," IEEE Trans. Power Electron., vol. 28, no. 5, pp. 2527-2538, May 2013.

[32] J. F. Pan, Y. Zou, N. Cheung, S. Member, and G. Cao, "On the voltage ripple reduction control of the linear switched reluctance generator for wave energy utilization," IEEE Trans. Power Electron., vol. 29, no. 10, pp. 5298-5307, 2014.

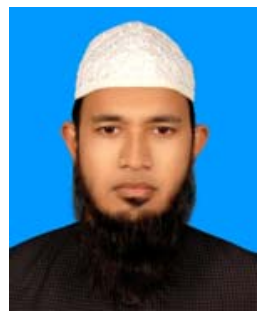

Omar Farrok (M'17) received B.Sc., M.Sc. and Ph.D. degree from Rajshahi University of Engineering and Technology (RUET), Rajshahi-6204, Bangladesh, in 2006, 2009, and 2016, respectively, all in the department of Electrical and Electronic Engineering (EEE). He was promoted to an Associate Professor in the department of EEE, Ahsanullah University of Science \& Technology (AUST), Dhaka-1208, Bangladesh.

$\mathrm{He}$ has authored and coauthored more than 30 technical papers. His research interest is in the field of electrical machine design, power electronics, and renewable energy technologies.

Dr. Farrok achieved the best paper award in ICEMS-2017, Sydney, Australia on 11-14 August 2017.

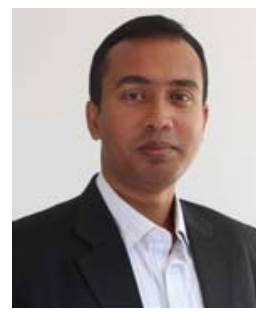

Md. Rabiul Islam (M'14-SM'16) received the B.Sc. and M.Sc. degree from Rajshahi University of Engineering and Technology (RUET), Rajshahi, Bangladesh, in 2003 and 2009, respectively; and the Ph.D. degree from University of Technology Sydney (UTS), Sydney, Australia, in 2014, all in electrical engineering.

He was appointed a lecturer at RUET in 2005 and promoted to full professor in 2017. In 2018 he joined the University of Wollongong, Australia, as a Research Fellow. From 2013 to 2014 , he was a Research Associate with UTS. His research interests are in the fields of power electronic converters, renewable energy technologies and smart grid.

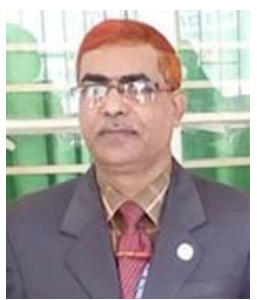

Md. Rafiqul Islam Sheikh (M'15) received the B. Sc. Eng. and M. Sc. Eng. degrees from Rajshahi University of Engineering \& Technology (RUET), Bangladesh, in 1992 and 2003, respectively, and $\mathrm{Ph}$. D. degree from Kitami Institute of Technology, Hokkaido, Kitami, Japan in 2010, all in Electrical Engineering.

$\mathrm{He}$ joined as a lecturer in EEE department RUET in 1994, where currently he is a Professor and Vice-Chancellor. His research interests are power system stability and renewable energy technologies. He has published many technical journal and conference papers, and authored or coauthored 3 books, and 3 book chapters.

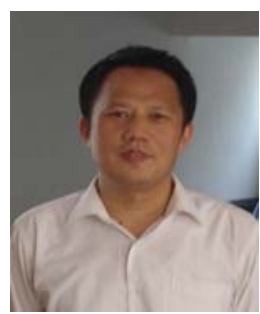

Youguang Guo (S'02-M'05-SM'06, IEEE) was born in Hubei, China in 1965. He received the B.E. degree from Huazhong University of Science and Technology, China in 1985, the M.E. degree from Zhejiang University, China in 1988, and the Ph.D. degree from University of Technology Sydney (UTS), Australia in 2004 all in electrical engineering. He is currently an associate professor with the School of Electrical and Data Engineering, UTS. His research areas include measurement and characterization of magnetic properties of magnetic materials, numerical analysis of electromagnetic field, electrical machine design and optimization, power electronic drives and motor control.

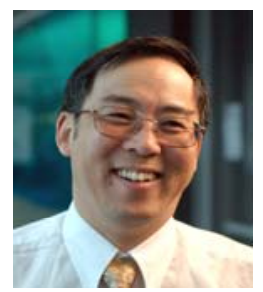

Jianguo Zhu (S'93-M'96-SM'03) received the B.E. degree in 1982 from Jiangsu Institute of Technology, Jiangsu, China, the M.E. degree in 1987 from Shanghai University of Technology, Shanghai, China, and the Ph.D. degree in 1995 from the University of Technology Sydney (UTS), Sydney, Australia, all in electrical engineering. He was appointed a lecturer at UTS in 1994 and promoted to full professor in 2004. In 2018, he joined the University of Sydney, Australia, as a full professor and Head of School for School of Electrical and Information Engineering. His research interests include computational electromagnetics, machines and drives, power electronics, renewable energy.

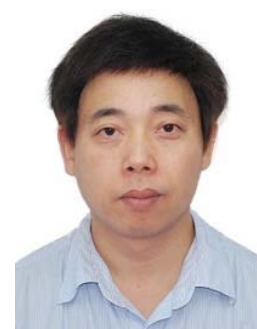

Gang Lei (M'14) received the B.S. degree in mathematics from Huanggang Normal University, China, in 2003, the M.S. degree in mathematics and Ph.D. degree in electrical engineering from Huazhong University of Science and Technology, China, in 2006 and 2009, respectively.

$\mathrm{He}$ is currently a lecturer at the University of Technology, Sydney, NSW, Australia. His current research interests include design optimization of electromagnetic devices and systems, including electrical machines and drive systems. 